\title{
Characterization of Pre- and Recession Periods for Indiana's Workforce
}

\author{
Kent Sellers \\ Department of Mathematical Science \\ Purdue University Fort Wayne \\ sellwk01@pfw . edu \\ Research and Analysis \\ Indiana Department of Workforce Development \\ wsellers@dwd.in.gov
}

\begin{abstract}
This paper studies the impact of The Great Recession period and the time period prior on the Indiana labor force. Specifically, it aims to show the impact across a variety of demographic, categorical variables (e.g. sex, age, and race)
\end{abstract}

\section{Introduction}

The United States have experienced six economic recessions. The Great Recession was the latest recession and lasted longer than any recession since World War II (Rich, 2013). There were many effects that the Great Recession had on the labor force. While some states were affected more than other, Indiana faced many hardships.

\subsection{Literature Review}

\subsubsection{How recessions impact unemployment}

"Early on, the nature of labor market adjustment displayed a notable resemblance to that observed in past severe downturns. During the latter half of 2009, however, the path of adjustment exhibited important departures from that seen during and after prior deep recessions. Recent data point to two warning signs going forward. First, the record rise in long-term unemployment may yield a persistent residue of long-term unemployed workers with weak search effectiveness. Second, conventional estimates suggest that the extension of Emergency Unemployment Compensation may have led to a modest increase in unemployment" (Elsby, Hobijn, and Sahin, 2010).

During recessions, the economy suffers. The long-run consequences (or "scarring) of recessions include: educational achievement, opportunity, private investment, and entrepreneurial activity and business formation (Irons, 2009).

One of the biggest lost opportunities are a result of job loss. Underlying trends appear in all recessions, the number of individuals that experience joblessness increases, along with an increased duration of joblessness. This can be costly for individuals and families. "In the most recent period (2001-03), about $35 \%$ of job losers are not employed at the subsequent survey date; about $13 \%$ re-employed full-time job losers are holding part-time jobs; full-time job losers who find new full-time jobs earn about $13 \%$ less on average on their new jobs than on the lost job..." (Farber,2005). Specifically, the Great Recession has been unique both in its depth and duration.

Unemployment and income loss can have long-term opportunity costs. "There is substantial evidence that economic outcomes are passed across generations. As such, economic hardships for parents will mean more economic hurdles for their children... For example, job loss and falling incomes can force 
families to delay or forgo a college education for their children" (Irons, 2009). The impact of job loss goes well beyond income and earnings, and can even impact one's mental health (Murphy and Athanasou, 1999).

\subsubsection{How recessions impact demographics}

Work has been done to examine how different types of workers in 17 middle-income countries were affected during The Great Recession. "Among active workers, youth experienced by far the largest adverse impacts on employment, unemployment, and wage employment, particularly relative to older adults. The percentage employment reductions, for example, were greatest for youth in each sector of the economy, as firms reacted to the shock by substituting away from inexperienced workers. Employment rates, as a share of the population, also plummeted for men. Larger drops in male employment were primarily attributable to men's higher initial rate of employment, although men's concentration in the hard-hit industrial sector also played an important role. Within each sector, percentage employment declines were similar for men and women. Added worker effects among women were mild, even among less-educated workers. Differences in labor market outcomes across education groups and urban or rural residence tended to be smaller"(Cho and Newhouse, 2011).

\subsubsection{How recessions impact Indiana's economy}

Indiana has a strong concentration in manufacturing, which has made the impacts of the Great Recession profound. "At the height of total unemployment in the state, more than 414,000 Hoosiers were without work at some time in the year, and almost one in four $(103,000)$ saw their extended benefits exhausted. In two years, the share of displaced workers who were unable to find employment prior to their benefits running out rose from 15 percent to 25 percent. While a portion of these workers may be simply unable to find work due to low demand, data on the industries from which unemployed workers previously worked paint a clear picture of structural unemployment. Manufacturing alone accounted for 10 percent of displaced workers, while the related industries of transportation, warehousing, and wholesale trade accounted for another 10 percent of displaced workers. Among those unemployed who lost benefits, a larger share of workers in manufacturing and related industries were represented" (Hicks, 2013).

By 2013, the recovery of the Great Recession in Indiana had shown that industries like health care, hospitality and food service were on the rise, while goods-producing industries like manufacturing and construction were down. These key industries that were hit led to the loss of many high-paying jobs. However, even though there are weaknesses within Indiana's economy, there are certainly competitive advantages if we can capitalize on the steady and balanced growth that Indiana has been experiencing (Kinghorn, 2014).

\subsection{Dataset}

Two primary sources of data were used: (1) IPUMS-CPS, University of Minnesota, www.ipums.org and (2) the monthly and annual state unemployment rates from the U.S. Bureau of Labor Statistics' Local Area Unemployment Statistics.

\subsubsection{IPUMS-CPS}

The Integrated Public Use Microdata Series, Current Population Survey (i.e. IPUMS-CPS), is data that comes from the Current Population Survey by the U.S. Bureau of Labor Statistics. The Minnesota Population Center at the University of Minnesota integrates this data over time and assigns uniform codes to variables. IPUMS is composed of microdata, meaning that each record corresponds to a person.

The IPUMS-CPS was the main source used to run the statistical analyses.

\subsubsection{LAUS}

The Local Area Unemployment Statistics (i.e. LAUS), is data from the U.S. Bureau of Labor Statistics and Indiana Department of Workforce Development. This data program is a federal-state partnership in which monthly estimates of total employment and unemployment are calculated for the many types of geographies. 


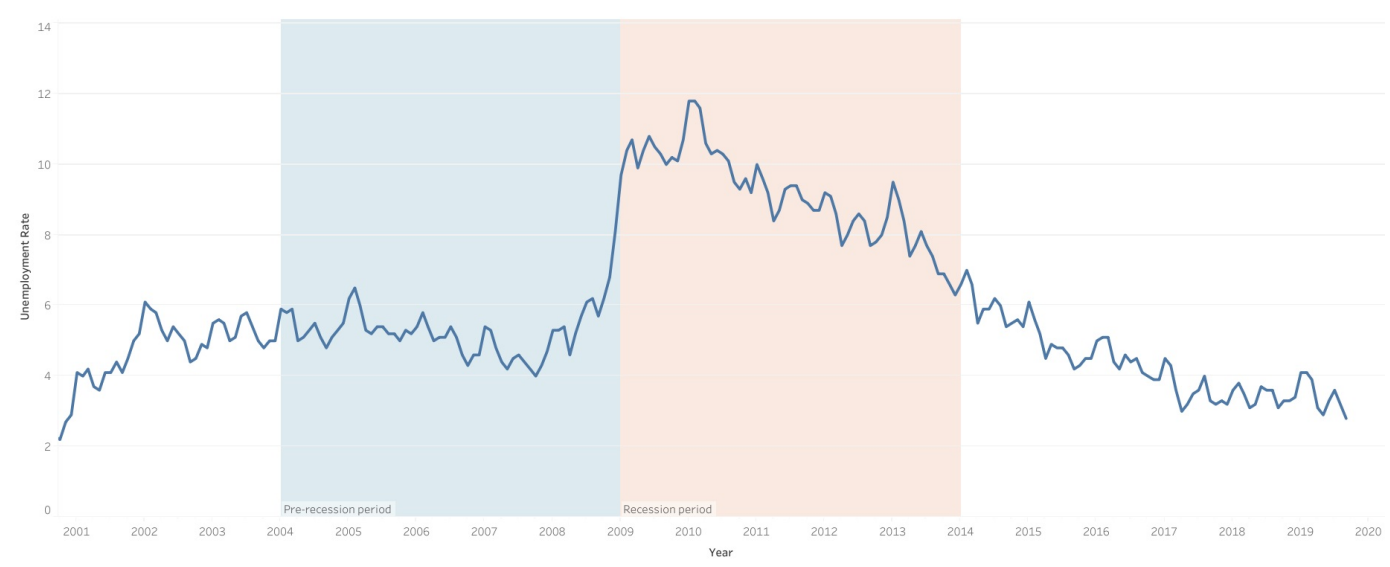

Figure 1: Indiana's monthly unemployment rate with reference periods, "pre-recession" and "recession".

LAUS monthly and annual rates for Indiana were used to determine the impact periods of the recession that were labeled as "pre-recession" and "recession." Figure 1 is a graph for Indiana to show how the annual unemployment rates have changed over the past couple decades, while showing how "pre-recession" and "recession" periods were calculated.

\subsection{Research question}

The impetus for this research was to better understand how economic recessions affect Indiana's labor force. The recession had many impacts, many of which revolved around the workforce. For the purposes of this study, the research question is as follows:

How can we characterize the "pre-recession" and "recession" periods for Indiana's labor force? Specifically, how are Indiana's working age population (18 and older) affected by The Great Recession across a variety of demographics?

Much of this analysis can be addressed in the IPUMS-CPS dataset. The Current Population Survey was a survey designed in the midst of the Great Depression, and a variety of labor force and demographic questions are asked to over a thousand households in Indiana.

Based on previous analysis on The Great Recession in the United States, a probable hypothesis would be that the demographics of workers most affected were young, male, lower education attainment, and ethnic minorities

\section{Methods}

Group analysis was the primary method used in this research. This characterization was done in two ways:

1. Populate sample counts of the categorical variables by the labor force status and fixing them on the different time periods (i.e. "pre-recession," "recession," and the combination of both periods)

2. Populate sample counts of the different time periods by the labor force status and fixing them on each label of categorical variables.

Re-coding certain labels within categorical variables were instrumental in having interpretive findings. Grouping labels into fewer labels made it easier to comprehend as well as working with larger sample sizes for each label.

With the populated samples of the labor force status, we can now see the labor force participation rate. Now that the counts and rates were populated, some basic grouped bar plots to help visualize the data 


\begin{tabular}{|c|c|c|c|c|c|c|c|c|c|}
\hline \multirow[b]{3}{*}{ Label } & \multicolumn{3}{|c|}{ Both Pre- and Recession Periods (2004- 2013) } & \multicolumn{3}{|c|}{ Pre-Recession Period (2004-2008) } & \multicolumn{3}{|c|}{ Recession Period (2009 - 2013) } \\
\hline & \multicolumn{2}{|c|}{ Not in Labor } & \multirow{2}{*}{$\begin{array}{c}x^{2} \\
p \text {-value }\end{array}$} & \multirow{2}{*}{$\begin{array}{c}\text { Not in Labor } \\
\text { Force }\end{array}$} & \multirow[b]{2}{*}{ In Labor Force } & \multirow{2}{*}{$\begin{array}{c}x^{2} \\
p \text {-value }\end{array}$} & \multirow{2}{*}{$\begin{array}{l}\text { Not in Labor } \\
\text { Force }\end{array}$} & \multirow{4}{*}{$\begin{array}{l}\text { In Labor Force } \\
31,186 \text { (7156) } \\
28,724 \text { (58\%6) }\end{array}$} & \multirow{4}{*}{$\begin{array}{c}x^{2} \\
p-v a l u e \\
<2.2 e-16\end{array}$} \\
\hline & & In Labor Force & & & & & & & \\
\hline Male & $25,027(2776)$ & $66,074(7396)$ & & $11,985(26 \% 6)$ & $34,888(7436)$ & & $13,042(2996)$ & & \\
\hline Female & $40,804(4096)$ & $60,396(6096)$ & $2.2 \mathrm{e}-16$ & $20,386(3996)$ & $31,672(6156)$ & $2.2 \mathrm{e}-16$ & $20,418(4296)$ & & \\
\hline College Age (18-24) & $6,819(3336)$ & $13,713(6796)$ & \multirow{4}{*}{$<2.2 \mathrm{e}-16$} & $3,248(30 \%)$ & $7,418(7056)$ & \multirow{4}{*}{$<2.2 \mathrm{e}-16$} & $3,571(36 \% 6)$ & $6,295(6496)$ & \multirow{4}{*}{$<2.2 \mathrm{e}-16$} \\
\hline Young Adult (25-44) & $10,917(17 \% 6)$ & $54,105(83 \%)$ & & $5,848(17 \%)$ & $29,360(8336)$ & & $5,069(1766)$ & $24,745(83 \%)$ & \\
\hline Older Adult (45-64) & $18,373(2666)$ & $52,605(7496)$ & & $9,008(25 \%)$ & $27,072(7596)$ & & $9,365(2796)$ & $25,533(7396)$ & \\
\hline Seniors $(65+)$ & $29,722(83 \% 6)$ & $6,047(1796)$ & & $14,267(8496)$ & $2,710(16 \%)$ & & $15,455(8236)$ & 3,337 (1836) & \\
\hline White only & $59,366(3496)$ & $114,915(6666)$ & \multirow{5}{*}{$5.32 \mathrm{E}-05$} & $29,291(33 \% 6)$ & $60,535(6736)$ & \multirow{5}{*}{0.0225} & $30,075(3666)$ & $54,380(6456)$ & \multirow{5}{*}{$1.10 E-06$} \\
\hline Black only & $5,153(36 \% 6)$ & $9,207(6496)$ & & $2,529(3496)$ & $4,893(6686)$ & & $2,624(3886)$ & $4,314(6256)$ & \\
\hline American Indiana/Aleut/Eskimo & $138(38 \% 6)$ & $228(6296)$ & & $49(2696)$ & $141(7486)$ & & $89(5196)$ & $87(4996)$ & \\
\hline Asian or Pacific Islander only & $664(35 \%)$ & $1,223(65 \%)$ & & $273(34 \% 6)$ & $538(6656)$ & & $391(36 \% 6)$ & $685(6496)$ & \\
\hline Two or more races & $510(36 \% 6)$ & $897(6496)$ & & $229(3486)$ & $453(66 \% 6)$ & & $281(3996)$ & $444(6196)$ & \\
\hline Not Hispanic & $63,902(3556)$ & $121,052(65 \% 6)$ & \multirow{2}{*}{$<2.2 \mathrm{e}-16$} & $31,519(33 \%)$ & $63,821(6796)$ & \multirow{2}{*}{$<2.2 \mathrm{e}-16$} & $32,383(3686)$ & $57,231(6456)$ & \multirow{2}{*}{$<2.2 e-16$} \\
\hline Hispanic & $1,929(2686)$ & $5,418(7496)$ & & $852(249 \%)$ & $2,739(766 \%)$ & & $1,077(2996)$ & $2,679(7196)$ & \\
\hline Citizen & $62,962(3456)$ & $119,835(6665)$ & \multirow{2}{*}{$<2.2 \mathrm{e}-16$} & $31,010(33 \% 6)$ & $63,223(6796)$ & \multirow[b]{2}{*}{$2.16 \mathrm{E}-\mathrm{O}$} & 31,952 (3656) & $56,612(6456)$ & \multirow[b]{2}{*}{$4.03 E-11$} \\
\hline Not acitizen & $2,869(3056)$ & $6,635(7096)$ & & $1,361(2996)$ & $3,337(7196)$ & & $1,508(3196)$ & $3,298(6996)$ & \\
\hline In metro area & $46,193(3496)$ & $88,634(6686)$ & 0,6996 & $22,205(33 \%)$ & $45,465(6796)$ & 0.3635 & $23,988(3686)$ & $43,169(64 \% 6)$ & $0237+2$ \\
\hline Not in metro area & $19,638(3496)$ & $37,836(66 \%)$ & 0.6996 & $10,166(3396)$ & $21,095(6736)$ & 0.3635 & $9,472(3666)$ & $16,741(6496)$ & 0.23 \\
\hline Married, spouse present & $34,827(3196)$ & $76,447(6966)$ & & $17,468(30 \% 6)$ & $41,110(7056)$ & & $17,359(3336)$ & $35,337(6776)$ & \\
\hline Married, spouse absent & $765(4096)$ & $1,130(6096)$ & & 345 (3796) & $593(6336)$ & & $420(4496)$ & $537(5686)$ & \\
\hline Separated & $775(28 \% 6)$ & $2,041(7296)$ & $<2.2 \mathrm{e}-16$ & $413(29 \%)$ & $1,016(7196)$ & $<2.2 \mathrm{e}-16$ & $362(2686)$ & $1,025(7486)$ & $<2.2 e-16$ \\
\hline Divorced & $7,462(31 \% 6)$ & 16,432 (6936) & & $3,363(28 \% 6)$ & $8,576(7256)$ & & $4,099(3496)$ & $7,856(6696)$ & \\
\hline Widowed & $10,632(8056)$ & $2,707(2096)$ & & $5,377(79 \% 6)$ & $1,394(2196)$ & & $5,255(8096)$ & $1,313(2096)$ & \\
\hline Never married/single & $11,370(2996)$ & $27,713(7196)$ & & $5,405(2886)$ & $13,871(7296)$ & & $5,965(3096)$ & $13,842(7036)$ & \\
\hline No service & $55,891(3336)$ & $115,912(67 \% 6)$ & $<22 \mathrm{e}-16$ & $27,438(3196)$ & $60,752(6996)$ & $22 e-16$ & $28,453(3496)$ & $55,160(6656)$ & $<22 \mathrm{e}-16$ \\
\hline Veteran & $9,940(4836)$ & $10,558(5296)$ & $2.20-10$ & $4,933(4696)$ & $5,808(54 \% 6)$ & $2.2=-10$ & $5,007(5196)$ & $4,750(4996)$ & $2.2 E-10$ \\
\hline No difficulty & $26,572(2996)$ & $64,783(7196)$ & $22 \mathrm{e}-$ & $2,521(2696)$ & $7,088(7456)$ & $22 e-1$ & $24,051(2996)$ & $57,695(7166)$ & $<22 e-16$ \\
\hline Has difficulty & $10,494(8156)$ & $2,533(1996)$ & $2.20-10$ & $1,085(83 \%)$ & $218(1776)$ & $2.2 e-10$ & $9,409(8196)$ & $2,215(1996)$ & $2.2 E-10$ \\
\hline Less than high school & $14,120(5666)$ & $11,097(4496)$ & & $7,629(55 \%)$ & $6,295(4596)$ & & $6,491(5796)$ & $4,802(4336)$ & \\
\hline High school graduate, or GED & $28,413(3886)$ & $46,484(6256)$ & & $13,757(3696)$ & $24,960(6456)$ & & $14,656(4096)$ & $21,554(6056)$ & \\
\hline Some college, no degree & $10,501(3196)$ & $23,703(6956)$ & & $5,018(29 \%)$ & $12,189(7196)$ & & $5,483(3296)$ & $11,514(68 \% 6)$ & \\
\hline Associate degree & $3,807(2296)$ & $13,197(7896)$ & $<2.2 \mathrm{e}-16$ & $1,766(20 \% 6)$ & $7,126(80 \% 6)$ & $<2.2 \mathrm{e}-16$ & $2,041(25 \% 6)$ & $6,071(7596)$ & $<2.2 \mathrm{e}-16$ \\
\hline Bachelors degree & $5,545(2196)$ & $20,741(7996)$ & & $2,652(21 \% 6)$ & $10,267(7966)$ & & $2,893(2296)$ & $10,474(7866)$ & \\
\hline Masters degree & $2,620(2436)$ & $8,087(7696)$ & & $1,162(2296)$ & $4,204(7866)$ & & $1,458(2776)$ & $3,883(73 \% 6)$ & \\
\hline Professional or doctoral degree & $825(2196)$ & $3,161(7996)$ & & $387(2056)$ & $1,549(80 \% 6)$ & & $438(2196)$ & $1,612(7996)$ & \\
\hline College or university full time & $2,860(5196)$ & $2,757(4996)$ & & $1,292(50 \% 6)$ & $1,308(5066)$ & & $1,568(5296)$ & $1,449(4886)$ & \\
\hline College or university part time & $149(15 \% 6)$ & $856(85 \% 6)$ & $<2.2 \mathrm{e}-16$ & $56(1396)$ & $390(8776)$ & $<2.2 \mathrm{e}-16$ & $93(1796)$ & $466(8336)$ & $<2.2 e-16$ \\
\hline Does not attend school, collegeo & 3,973 (1996) & $17,019(8196)$ & & 1,203 (19\%) & $5,211(8196)$ & & 2,770 (1996) & $11,808(8156)$ & \\
\hline
\end{tabular}

Figure 2: Categorical variable by labor force status fixed on time period

were supportive in discovering those differences. In order to determine whether those differences were significant or due to random variation, chi-squared $\left(\chi^{2}\right)$ tests were performed across all the groups within both characterizations.

\section{Results}

The following tables reflect the results of the group analysis that was done. Similar to Figure 1, the colors in the table reflect "pre-recession" and "recession" periods. A darker salmon color was used to reflect where the $p$ value of the chi-squared tests were greater than 0.05 .

As seen below, many of the chi-squared tests did result in low $\mathrm{p}$ values signifying that most of the differences were not due to random variation.

Figure 2 is a table that characterizes the categorical variables by the labor force status, while being fixed on the time periods:

Figure 3 is a table that characterizes the time periods by the labor force status, while being fixed on each label of a categorical variable:

\section{Discussion}

\subsection{Insights}

The age group with the largest negative effect on the labor force during The Great Recession were college age (18 to 24 years). This would make sense given that there is a positive relationship between unemployment rates and college enrollment (Long, 2004).

Despite there being less opportunities in the labor market, the only age group that were found to increase their labor force participation rate were seniors (65 years and older). Again, this would seem to directly correlate to the fact the retirement portfolios took a hit as the stock prices fell during the 


\begin{tabular}{|c|c|c|c|c|}
\hline Label & Time Period & Not in labor force & In labor force & $\begin{array}{c}\chi^{2} \\
p \text {-value }\end{array}$ \\
\hline \multirow{2}{*}{ Male } & Pre-Recession Period (2004 - 2008) & $11,985(26 \%)$ & $34,888(74 \%)$ & \multirow{2}{*}{$<2.2 \mathrm{e}-16$} \\
\hline & Recession Period (2009 - 2013) & $13,042(29 \%)$ & $31,186(71 \%)$ & \\
\hline \multirow{2}{*}{ Female } & Pre-Recession Period (2004 - 2008) & $20,386(39 \%)$ & $31,672(61 \%)$ & \multirow{2}{*}{$1.02 \mathrm{E}-14$} \\
\hline & Recession Period (2009-2013) & $20,418(42 \%)$ & $28,724(58 \%)$ & \\
\hline \multirow{2}{*}{ College Age (18-24) } & Pre-Recession Period (2004 - 2008) & $3,248(30 \%)$ & $7,418(70 \%)$ & \multirow{2}{*}{$<2.2 \mathrm{e}-16$} \\
\hline & Recession Period (2009 - 2013) & $3,571(36 \%)$ & $6,295(64 \%)$ & \\
\hline \multirow{2}{*}{ Young Adult (25-44) } & Pre-Recession Period (2004 - 2008) & $5,848(17 \%)$ & $29,360(83 \%)$ & \multirow{2}{*}{0.1859} \\
\hline & Recession Period (2009-2013) & $5,069(17 \%)$ & $24,745(83 \%)$ & \\
\hline \multirow{2}{*}{ Older Adult (45-64) } & Pre-Recession Period (2004 - 2008) & $9,008(25 \%)$ & $27,072(75 \%)$ & $1.40 F_{-}-08$ \\
\hline & Recession Period (2009 - 2013) & $9,365(27 \%)$ & $25,533(73 \%)$ & $1.405-60$ \\
\hline Seniors $(65+)$ & Pre-Recession Period (2004 - 2008) & $14,267(84 \%)$ & $2,710(16 \%)$ & $653 F_{-} 06$ \\
\hline 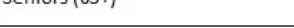 & Recession Period (2009 - 2013) & $15,455(82 \%)$ & $3,337(18 \%)$ & 0.00 \\
\hline White only & Pre-Recession Period (2004 - 2008) & $29,291(33 \%)$ & $60,535(67 \%)$ & $<2,2 e-16$ \\
\hline White oniy & Recession Period (2009 - 2013) & $30,075(36 \%)$ & $54,380(64 \%)$ & $<2.2 \mathrm{e}-10$ \\
\hline & Pre-Recession Period (2004 - 2008) & $2,529(34 \%)$ & $4,893(66 \%)$ & \\
\hline Black only & Recession Period (2009 - 2013) & $2,624(38 \%)$ & $4,314(62 \%)$ & 3.17E-06 \\
\hline AmericanIndiana/Aleut/Eskimo & Pre-Recession Period (2004 - 2008) & $49(26 \%)$ & $141(74 \%)$ & $176 \mathrm{E}-06$ \\
\hline 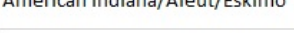 & Recession Period (2009 - 2013) & $89(51 \%)$ & $87(49 \%)$ & $1.70 \mathrm{E}-00$ \\
\hline Asjan or Pacificlslander only & Pre-Recession Period (2004 - 2008) & $273(34 \%)$ & $538(66 \%)$ & 0.2475 \\
\hline Astan or pacinc istander oniy & Recession Period (2009 - 2013) & $391(36 \%)$ & $685(64 \%)$ & $0.24 / 3$ \\
\hline Tweormore races & Pre-Recession Period (2004 - 2008) & $229(34 \%)$ & $453(66 \%)$ & 004943 \\
\hline IWo or more races & Recession Period (2009-2013) & $281(39 \%)$ & $444(61 \%)$ & 0.04943 \\
\hline Not Hisnanic. & Pre-Recession Period (2004 - 2008) & $31,519(33 \%)$ & $63,821(67 \%)$ & $<220-16$ \\
\hline 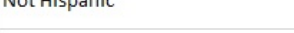 & Recession Period (2009 - 2013) & $32,383(36 \%)$ & $57,231(64 \%)$ & $<2.2 e-10$ \\
\hline Hispanic & Pre-Recession Period (2004 - 2008) & $852(24 \%)$ & $2,739(76 \%)$ & $165 F_{-} 06$ \\
\hline Hispanic & Recession Period (2009 - 2013) & $1,077(29 \%)$ & $2,679(71 \%)$ & $1.005-00$ \\
\hline Citizen & Pre-Recession Period (2004 - 2008) & $31,010(33 \%)$ & $63,223(67 \%)$ & $<2.2 e-16$ \\
\hline Citizen & Recession Period (2009-2013) & $31,952(36 \%)$ & $56,612(64 \%)$ & $2.2 e-10$ \\
\hline Not a citizen & Pre-Recession Period (2004 - 2008) & $1,361(29 \%)$ & $3,337(71 \%)$ & 0,01128 \\
\hline & Recession Period (2009-2013) & $1,508(31 \%)$ & $3,298(69 \%)$ & \\
\hline In metro area & Pre-Recession Period (2004 - 2008) & $22,205(33 \%)$ & $45,465(67 \%)$ & $2.2 \mathrm{e}-16$ \\
\hline In metro area & Recession Period (2009 - 2013) & $23,988(36 \%)$ & $43,169(64 \%)$ & $2.2 \mathrm{e}-10$ \\
\hline Notin metro area & Pre-Recession Period (2004 - 2008) & $10,166(33 \%)$ & $21,095(67 \%)$ & $<220-16$ \\
\hline Fot in metro dred & Recession Period (2009-2013) & $9,472(36 \%)$ & $16,741(64 \%)$ & $<2.2 e-10$ \\
\hline Married spouse present & Pre-Recession Period (2004 - 2008) & $17,468(30 \%)$ & $41,110(70 \%)$ & $e-16$ \\
\hline & Recession Period (2009-2013) & $17,359(33 \%)$ & $35,337(67 \%)$ & \\
\hline Married, spouse absent & Pre-Recession Period (2004 - 2008) & $345(37 \%)$ & $593(63 \%)$ & 0.001898 \\
\hline TVarrlea, spouse absent & Recession Period (2009 - 2013) & $420(44 \%)$ & $537(56 \%)$ & 0.001898 \\
\hline Senarated & Pre-Recession Period (2004 - 2008) & $413(29 \%)$ & $1,016(71 \%)$ & 0.1048 \\
\hline Separdeu & Recession Period (2009-2013) & $362(26 \%)$ & $1,025(74 \%)$ & \\
\hline Divorced & Pre-Recession Period (2004 - 2008) & $3,363(28 \%)$ & $8,576(72 \%)$ & $<2.2 \mathrm{e}-16$ \\
\hline & Recession Period (2009-2013) & $4,099(34 \%)$ & $7,856(66 \%)$ & \\
\hline Widowed & Pre-Recession Period (2004 - 2008) & $5,377(79 \%)$ & $1,394(21 \%)$ & 0.4035 \\
\hline Widowed & Recession Period (2009-2013) & $5,255(80 \%)$ & $1,313(20 \%)$ & 0.4035 \\
\hline Nevermarried/single & Pre-Recession Period (2004 - 2008) & $5,405(28 \%)$ & $13,871(72 \%)$ & $6.62 F_{-}-06$ \\
\hline & Recession Period (2009-2013) & $5,965(30 \%)$ & $13,842(70 \%)$ & \\
\hline No service & Pre-Recession Period (2004 - 2008) & $27,438(31 \%)$ & $60,752(69 \%)$ & $<2.2 \mathrm{e}-16$ \\
\hline & Recession Period (2009 - 2013) & $28,453(34 \%)$ & $55,160(66 \%)$ & \\
\hline Veteran & Pre-Recession Period (2004 - 2008) & $4,933(46 \%)$ & $5,808(54 \%)$ & $138 \mathrm{~F}-14$ \\
\hline & Recession Period (2009-2013) & $5,007(51 \%)$ & $4,750(49 \%)$ & \\
\hline No difficulty & Pre-Recession Period (2004 - 2008) & $2,521(26 \%)$ & $7,088(74 \%)$ & $8.44 F_{-11}$ \\
\hline & Recession Period (2009-2013) & $24,051(29 \%)$ & $57,695(71 \%)$ & \\
\hline Has difficulty & Pre-Recession Period (2004 - 2008) & $1,085(83 \%)$ & $218(17 \%)$ & 0.001413 \\
\hline & Recession Period (2009-2013) & $9,409(81 \%)$ & $2,215(19 \%)$ & \\
\hline Jessthan high scheol & Pre-Recession Period (2004 - 2008) & $7,629(55 \%)$ & $6,295(45 \%)$ & $202 F_{-}-05$ \\
\hline Less than nign scriool & Recession Period (2009-2013) & $6,491(57 \%)$ & $4,802(43 \%)$ & \\
\hline High school graduate, or GED & Pre-Recession Period (2004 - 2008) & $13,757(36 \%)$ & $24,960(64 \%)$ & $<220-16$ \\
\hline & Recession Period (2009 - 2013) & $14,656(40 \%)$ & $21,554(60 \%)$ & \\
\hline Some college, no degree & Pre-Recession Period (2004 - 2008) & $5,018(29 \%)$ & $12,189(71 \%)$ & $E-10$ \\
\hline & Recession Period (2009 - 2013) & $5,483(32 \%)$ & $11,514(68 \%)$ & \\
\hline Associate degree & Pre-Recession Period (2004 - 2008) & $1,766(20 \%)$ & $7,126(80 \%)$ & $<2.2 \mathrm{e}-16$ \\
\hline & Recession Period (2009-2013) & $2,041(25 \%)$ & $6,071(75 \%)$ & \\
\hline Bachelors degree & Pre-Recession Period (2004 - 2008) & $2,652(21 \%)$ & $10,267(79 \%)$ & 0.02781 \\
\hline & Recession Period (2009 - 2013) & $2,893(22 \%)$ & $10,474(78 \%)$ & \\
\hline degree & Pre-Recession Period (2004 - 2008) & $1,162(22 \%)$ & $4,204(78 \%)$ & -11 \\
\hline & Recession Period (2009 - 2013) & $1,458(27 \%)$ & $3,883(73 \%)$ & \\
\hline Professional or doctoral degr & Pre-Recession Period (2004 - 2008) & $387(20 \%)$ & $1,549(80 \%)$ & 0.3017 \\
\hline & Recession Period (2009-2013) & $438(21 \%)$ & $1,612(79 \%)$ & \\
\hline College or university full time & Pre-Recession Period (2004 - 2008) & $1,292(50 \%)$ & $1,308(50 \%)$ & 009345 \\
\hline 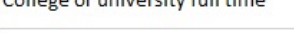 & Recession Period (2009 - 2013) & $1,568(52 \%)$ & $1,449(48 \%)$ & \\
\hline or university part time & Pre-Recession Period (2004 - 2008) & $56(13 \%)$ & $390(87 \%)$ & 0.08554 \\
\hline . & Recession Period (2009 - 2013) & $93(17 \%)$ & $466(83 \%)$ & 0.08534 \\
\hline Does not attend school, college & Pre-Recession Period (2004 - 2008) & $1,203(19 \%)$ & $5,211(81 \%)$ & 0.6899 \\
\hline or university & Recession Period (2009 - 2013) & $2,770(19 \%)$ & $11,808(81 \%)$ & \\
\hline
\end{tabular}

Figure 3: time period by labor force status fixed on an individual label of a categorical variable 
financial crisis. "Stock prices fell roughly 50 percent from peak to trough from October 2007 to March 2009. These drops in stock prices are large relative to those associated with earlier recessions since World War II" (Dwyer, 2009).

Following national trends mentioned earlier, Hoosier men and women were affected by the recession at a similar rate in the labor force.

Metro areas also seemed to be affected at a similar rate. However, the differences between metro status and labor force status had high $\mathrm{p}$ values signifying a good chance that the differences were due to random variation. However, when looking at the differences between labor force status and time period fixed on the metro status label, the $\mathrm{p}$ values were low. Although one can't say with certainty that the metro areas were affected the same, it would interesting to follow up in that topic due to the fact that typically communities will attempt to diversify through many industries in order to lessen the impacts of a recession. In Indiana, many of the metro areas have diversified industries, while the rural areas are heavily concentrated in manufacturing and agriculture. Are the affects that recessions have on the labor force the same in Indiana? Or are communities lessening other effects of a recession?

Although those that have any difficulty (i.e. disabled) is a demographic that has some of the lowest labor force participation, their participation rate in Indiana increased during a recession. It would be interesting to do more research to find out why this is, and what disabilities are affected the same. This can be done within the IPUMS-CPS dataset, but one would have to either re-code the variables to make sure one isn't double counting individuals that have multiple disabilities, or you would have to study the disabilities themselves rather than the connection to the individual.

\subsection{Limitations}

The biggest limitation of the analysis is a repeated measurement error. It was not taken into consideration that the same person could take the survey more than once. In the CPS, a household that receives the survey will take the survey for four consecutive months, then will be off for 8 consecutive months, and then will take again for another four consecutive months.

\subsection{Future Analysis}

The first thing that would need to be done, would be to restrict the repeated measurement error as described in the limitations section. One possible way to get around this error is to create a unique identifier for each person within the IPUMS-CPS data by combining the "SERIAL" label (which identifies a unique household) and the "PERNUM" label (which numbers the persons within each household). Then one can restrict the dataset with the first time the unique identifier for an individual appears.

Where there were unexpected high $\mathrm{p}$ values, one could apply more advanced statistical techniques, especially where $\mathrm{p}$ values were unexpectedly high, such as part of the metro status tests.

In future work, it would be interesting to do predictive analysis by using the categorical variables and labor force status to determine if one is in a recession period.

As previously mentioned, it could be enlightening to do some future analysis as to why metro and not metro areas seemed to be affected the same. Also, one could research why individuals with difficulties increased in the labor force, and how those different disabilities are affected.

\section{References}

[1] Rich, R. (2013). The Great Recession. Federal Reserve Board at https://www . federalreservehistory. org/essays/great_recession_of_200709

[2] Elsby, M., Hobijn, B., and Sahin, A. (2010). The Labor Market in the Great Recession. Retrieved from https://www.nber.org/papers/w15979.pdf

[3] Irons, J. (2009). Economic scarring: The long-term impacts of the recession. Economic Policy Institute, at https://www.epi.org/publication/bp243/ 
[4] Farber, Henry S. (2005). What Do We Know about Job Loss in the United States? Evidence from the Displaced Workers Survey, 1984-2004. Working paper \#498, Princeton University, 2005, at http://www . irs princeton.edu/pubs/pdfs/498.pdf

[5] Murphy, G.C., and Athanasou, J.A. (1999). The effect of unemployment on mental health. Journal of Occupational and Organizational Psychology. Vol. 72, pp. 83-99.

[6] Cho Y. and Newhouse, D. (2011). How Did the Great Recession Affect Different Types of Workers? Evidence from 17 Middle-Income Countries. The World Bank, at https://openknowledge.worldbank. org/bitstream/handle/10986/3401/WPS5636.pdf?sequence=1\&isAllowed=y

[7] Hicks, M.J. (2013). Labor Markets After The Great Recession: Unemployment and Policy for Indiana Ball State University Center for Business and Economic Research, at https://projects.cberdata.org/ reports/StructuralUnemp.pdf

[8] Kinghorn, M.R. (2014). Indiana Jobs: Recession, Recovery. Indiana Business Review, at https://www ibrc.indiana.edu/ibr/2014/fall/article1.html

[9] Long, B.T. (2004). How Have College Decisions Changed Overtime? An Application of the Conditional Logistic Choice Model.” Journal of Econometrics 121 (1-2): pp. 271-96.

[10] Dwyer, G.P. (2009). Stock Prices in the Financial Crisis: Notes from the Vault. Federal Reserve Bank of Atlanta, at https://www.frbatlanta.org/cenfis/publications/notesfromthevault/0909.aspx 